\title{
Novel Image-Based 3D Reconstruction of the Human Jaw using Shape from Shading and Feature Descriptors
}

\author{
Aly S. Abdelrahim ${ }^{1}$ \\ asabde02@louisville.edu \\ Mostafa A. Abdelrahman ${ }^{1}$ \\ maabde01@louisville.edu \\ Hossam Abdelmunim ${ }^{12}$ \\ hossameldin.hassan@eng.asu.edu.eg \\ Aly A. Farag ${ }^{1}$ \\ http://www.cvip.uofl.edu \\ M. Miller ${ }^{1}$ \\ http://www.cvip.uofl.edu
}

${ }^{1}$ Department of Electrical and Computer

Engineering, University of Louisville,

Louisville, KY, 40292, USA.

${ }^{2}$ Computer and Systems Engineering

Department, Faculty of Engineering,

Ain Shams University,

Cairo 11517, Egypt.

\begin{abstract}
In this paper, we propose a novel approach for 3D surface reconstruction of the human jaw. Due to the difficulties of setting up a data acquisition system inside the mouth, we use an intra-oral camera to capture a sequence of calibrated images. These images are registered together to build a panoramic view of the jaw. We incorporate a shape from shading(SFS) algorithm that benefits from camera calibration parameters to build a 3D model from the panoramic image obtained from the previous stage. Our approach results in a 3D surface which has more fine details compared with those resulting from other literature techniques. We will demonstrate different artificial jaws surfaces reconstruction to show the efficiency of our system.
\end{abstract}

\section{Introduction}

Dentistry requires accurate 3-D representation of the teeth and jaw for diagnostic and treatment purposes. For example, orthodontic treatment involves the application, over time, of force systems to teeth to correct malocclusion. In order to evaluate tooth movement progress, the orthodontist monitors this movement by means of visual inspection, intra-oral measurements, fabrication of casts, photographs, and radiographs; this process is both costly and time consuming. Moreover, repeated acquisition of radiographs may result in unknown effects. Obtaining a cast of the jaw is a complex operation for the dentist, an unpleasant experience for the patient, and also may not provide all the necessary details of the jaw [ $[2]$ ].

Oral and maxillofacial radiology can provide the dentist with abundant 3-D information of the jaw. Current and evolving methods include computed tomography (CT), tomosynthesis [四, tuned-aperture CT (TACT) [四, and localized, or "cone-beam," computed tomography $[\mathrm{Q}]$. While oral and maxillofacial radiology is now widely accepted as a routine 
technique for dental examinations, the equipment is rather expensive and the resolution is frequently too low for 3-D modeling of dental structures. Furthermore, the radiation dose required to enhance both contrast and spatial resolution can be unacceptably high.

Substantial efforts have focused recently on computerized diagnosis in dentistry [ ${ }^{0}$ ]. Bernard et. al. [日] developed an expert system where cephalometric measurements are acquired manually from the analysis of radiographs and plaster models. Laurendeau et al. [घ] presented a computer-vision technique for the acquisition of jaw data from inexpensive dental wafers. That system was capable of obtaining imprints of the teeth. Usually, most of the 3-D systems for dental applications found in the literature rely on obtaining an intermediate solid model of the jaw (cast or teeth imprints) and then capturing the 3-D information from that model. User interaction is needed in such systems to determine the 3-D coordinates of fiducial reference points on a dental cast. Other systems that can measure the 3-D coordinates have been developed using either mechanical contact [ $[$ ] or a travelling light principle [ $[$ ] [ $[$ ]. Goshtasby et al. [ $[$ ] designed a range scanner based on white light to reconstruct the cast. The scanner used the subtractive light principle to create very thin shadow profiles on the cast.

In this paper, we use an intra-oral camera to capture a sequence of calibrated images which is more comfortable to patients. Also it does not require a long time to scan a jaw. The resulting sequence of images covers the jaw and contains overlapped image regions. These sequential images are taken in pairs to perform image alignment by estimating projective transformations. This process incorporates points correspondences accurately found by the affine and scale invariant transformation approach (known as ASIFT). After estimating the projective transformations, the image pairs are used together in order to build a panoramic image of the whole jaw. The new view is used to build a 3D surface using the shape from shading (SFS) algorithm. The used SFS is depending on calibration parameters. We use a formulation of the SFS that uses intrinsic and extrinsic camera parameters such that we obtain a better surface. This technique is compared with former approaches and the difference is significant. Incorporating camera parameters results in surfaces with fine details that can not be handled by the other methods that involve SFS. Different 3D reconstruction results of artificial jaws will be demonstrated to show the efficiency of the proposed technique.

\section{Data Acquisition}

The human jaw is not a friendly environment for calibrated video measurements. There are problems with lighting (effect of saliva, tooth discolorization, gum texture, and other sources of specularity), motion (even inevitable slight motions of the upper/lower jaw may lead to errors far beyond the desired tolerance of submillimeters accuracy), and data acquisition time (it is not comfortable to open the jaw widely for over a minute or two). There are small cameras available that can provide viewing of the human jaw. The lenses are usually active and allocated on the dental probe to provide viewing not calibrated image capturing. Hence, a major problem to overcome would be the design of a small size CCD camera with passive or active lenses that can be calibrated with respect to a reference frame. In this paper we used an intra-oral camera with an attached small light source to acquire 2-D images of the teeth. Some specifications are given as: LED light source, 1/4" CCD Sensor; 320,000 pixels $\left(\mathrm{PAL}^{1}\right), 270,000$ pixels $\left(\mathrm{NTSC}^{2}\right)$ and resolution $512 \times 582(\mathrm{PAL}) ; 512 \times 942(\mathrm{NTSC})$. The

\footnotetext{
${ }^{1}$ Phase Alternating Line

${ }^{2}$ National Television System Committee.
} 
objective of this part is to remove global differences between two given images (source and target). Every two sequential images will be aligned together. The output of this process is a panoramic image resulting from putting (stitching) the aligned sequence of images for the whole jaw. The description of registering two images by mapping points correspondences is given in this section. A point in the source image is related to its corresponding target position by a projective transformation [四]. Note that the overlap between the two images results from imaging the same object by the camera to a different positions. Given a set of $K$ homogeneous image point correspondences $\mathbf{C}_{o}^{s} \in R^{3}$ (in the source image) and $\mathbf{C}_{o}^{t} \in R^{3}$ (in the target) where $o \in 1,2, \ldots, K$ : we want to estimate a projective transformation $\mathbf{H}_{3 \times 3}$ to map a source image point to its corresponding target image position by the relation $s_{o} \mathbf{C}_{o}^{t}=\mathbf{H C}_{o}^{s}$ where $s_{o}$ is a scaling coefficient for the projective transformation effect. Actually, this equation will lead to two linear equations: $h_{11} x_{o}^{s}+h_{12} y_{o}^{s}+h_{13}-h_{31} x_{o}^{t} x_{o}^{s}-h_{32} x_{o}^{t} y_{i}^{s}-h_{33} x_{o}^{t}=0$ and $h_{21} x_{o}^{s}+h_{22} y_{o}^{s}+h_{23}-h_{31} y_{o}^{t} x_{o}^{s}-h_{32} y_{o}^{t} y_{o}^{s}-h_{33} y_{o}^{t}=0$ where $x$ and $y$ are the coordinates of point. We can construct a linear system of equations $\Psi \Theta=\mathbf{0}$ where $\Theta=\left[h_{11} h_{12} \ldots h_{33}\right]$ is the parameters column of the projective transformation $\mathbf{H}$ and $\Psi$ is the coefficients matrix. We estimate the projective transformation parameters by solving this linear system through the singular value decomposition approach. If the points correspondences are accurate, the overall difference between the source and target images vanishes when they are fused together. The success of this process is highly dependent on selecting the source and target points correspondences.

\section{Affine Scale-Invariant Feature Transform(ASIFT)}

The ASIFT [ $\square]$ is one of the most successful and promising approaches in the literature to find point correspondences. We used this technique to find accurately these points and hence obtain perfect alignment.

The resulting matched points from the ASIFT algorithm may include incorrectly matched points so RANSAC [ $[$ ] is used to fit a transformation model to those points. A random sample consists of four points which is the minimal subset of the interest points sufficient to determine a projective transformation is selected. The transformation is estimated using the algorithm described above with the chosen random sample, then the resulting transformation is evaluated using all correspondence points available. These points which deviate from the current transformation model by a specified threshold are considered outliers, hence the support of the model will be measured by the ratio of the inlier to the total number of points. This procedure is repeated M-times, where $M \leq \frac{N !}{2 ! .(N-2) !}$ then the best fit transformation model is the one with the maximum support, and the the points marked as outliers are excluded from the interest points.

\section{Shape from Shading using a Calibrated Image}

Among the tools used in shape extraction from a single view is the shape from shading (SFS) technique. SFS has been primarily studied by Horn [ $[\mathbb{}]$ and his colleagues at MIT. There have been many developments in the algorithms (e.g., [四], [四], [四]). SFS assumes that the surface orientation at a point $\mathbf{P}$ on a surface $\mathbf{S}$ is determined by the unit vector perpendicular to the plane tangent to $S$ at $P$. Under the assumption of orthographic projections, the elemental change in the depth $Z$ at an image point $(x, y)$ can be expressed as $\delta z \approx \frac{\partial Z}{\partial x} \delta x+\frac{\partial Z}{\partial y} \delta y$. 
The partial derivatives are called surface gradients $(\mathbf{r}, \mathbf{q})$. The normal to a surface patch is related to the surface gradient by $\mathbf{n}=\mathbf{r} \times \mathbf{q}$.Assuming that surface patches are homogeneous and uniformly lit by distant light sources, the brightness $E(x, y)$ seen at the image plane often depends only on the orientation of the surface. This dependence of brightness on surface orientation can be represented as a function $R($.$) defined on the Gaussian sphere. Thus, the SFS$ problem is formulated as finding a solution to the brightness equation: $E(x, y)=R(\mathbf{r}, \mathbf{q}, \mathbf{L})$, where $R(\mathbf{r}, \mathbf{q}, \mathbf{L})$ is the surface reflectance map and $\mathbf{L}$ is the illuminate direction.

A number of algorithms were developed to estimate the illuminant direction (e.g., []). Because the white light beam in our design is built in the CCD camera, the assumption that the illuminant direction is known beforehand is valid. However, the assumption of orthographic projection is not adequate for the dental application because the camera is very close to the object. Some SFS approaches using perspective projection were found in the literature (e.g. [ $[\square]$, [ $[\mathbb{\square}]$ ). However, most of these approaches ignore the camera extrinsic parameters, hence cannot provide metric information of the depth. In our approach, the CCD camera is calibrated and the camera parameters are used in the SFS algorithm to obtain a metric representation of the teeth and gum surfaces. To calibrate the camera, the relation between the $3 \mathrm{D}$ point $\mathbf{P}=(X, Y, Z)^{T}$ and the corresponding image coordinates $\mathbf{p}=(x, y)^{T}$ is written as; $s \overrightarrow{\mathbf{p}}=\mathbf{M} \overrightarrow{\mathbf{P}}$ where $s$ is a scalar, $\mathbf{p}$ and $\mathbf{P}$ are the extended vectors $\left[\mathbf{p}^{T} 1\right]^{T}\left[\mathbf{P}^{T} 1\right]^{T}$, and $\mathbf{M}$ is called the camera calibration matrix. In general, $\mathbf{M}=\mathbf{K}[\mathbf{Q}, \mathbf{T}]$ where $\mathbf{K}$ is a matrix containing all the camera intrinsic parameters and $\mathbf{Q}, \mathbf{T}$ are the rotation matrix and the translation vector respectively. The matrix $\mathbf{M}$ has 12 elements but has only 11 degrees of freedom because it is defined up to a scale factor (e.g., [四]).

The standard method of calibration is to use an object with known size and shape and extract the reference points from the object image. It can be shown that given $N$ points $(N \geq 6)$ in general positions, the camera can be calibrated [四]. The perspective projection matrix $\mathbf{M}$ can be decomposed as [ $\mathbf{B b}]$ where $\mathbf{B}$ is a $3 \times 3$ matrix and $\mathbf{b}$ is a $3 \times 1$ vector such that;

$$
s \overrightarrow{\mathbf{p}}=\mathbf{B P}+\mathbf{b} \quad \text {, or } \mathbf{P}=\mathbf{B}^{-1}(s \overrightarrow{\mathbf{p}}-\mathbf{b})=f(s(x, y))
$$

This last equation represents a line in the 3D space corresponding to the visual ray passing through the optical center and the projected point $\mathbf{p}$. By finding the scalar s, $f(s(x, y))$ will define a unique 3D point $\mathbf{P}$ on the object. The surface normal at $\mathbf{P}$ is defined to be the cross product of the two gradient vectors $\mathbf{r}=\frac{d f(s(x, y))}{d x}, \mathbf{q}=\frac{d f(s(x, y))}{d y}$ The surface reflectance $R($.$) becomes a function of the scalar s$ defined in Eq.(1), i.e.,

$$
R(s)=\frac{(\mathbf{r} \times \mathbf{q}) \cdot \mathbf{L}}{|\mathbf{r} \times \mathbf{q}||\mathbf{L}|}
$$

The formulation of the SFS problem becomes finding the scalar $s$ that solves the brightness equation $g(s)=E(x, y)-R(s)=0$. This can be solved using a Taylor's series expansion and applying the Jacoby iterative method [四]. After $n$ iterations, for each point $(x, y)$ in the image, $s_{x, y}^{n}$ is given as follows :

$$
s_{x, y}^{n}=s_{x, y}^{n-1}+\frac{-g\left(s_{x, y}^{n-1}\right)}{\frac{d}{d s_{x, y}} g\left(s_{x, y}^{n-1}\right)}
$$


where,

$$
\begin{gathered}
\frac{d}{d s_{x, y}} g\left(s_{x, y}^{n-1}\right)=-\frac{d \mathbf{N}}{d s_{x, y}} \cdot \frac{\mathbf{L}}{|\mathbf{L}|} \\
\frac{d \mathbf{N}}{d s_{x, y}}=-\frac{d \mathbf{v}}{d s_{x, y}} \frac{1}{\sqrt{\left(\mathbf{v}^{t} \mathbf{v}\right)^{3}}}\left(\mathbf{v}^{t} \frac{d \mathbf{v}}{d s_{x, y}}\right) \\
\frac{d \mathbf{v}}{d s_{x, y}}=\mathbf{B}^{-1} \overrightarrow{\mathbf{p}} \times \mathbf{B}^{-1}\left(0, s_{x, y-1,0}\right)^{t}+\mathbf{B}^{-1}\left(0, s_{x, y-1,0}\right)^{t} \times \mathbf{B}^{-1} \overrightarrow{\mathbf{p}}
\end{gathered}
$$

where $\mathbf{v}=\mathbf{r} \times \mathbf{q}$

\section{Shape from Shading using Partial differential Equation(PDE)}

In general, the brightness of a surface patch depends on its orientation relative to both the light source and the viewer [四]. Under the simplifying assumption that the viewer and the light source are far from the object, the image irradiance equation can be written as follows:

$$
\mathbf{E}_{o}(x)=\mathbf{R}_{o}\left(\hat{\mathbf{n}}_{o}(x)\right)
$$

where $\mathbf{E}_{o}(x)$ is the image irradiance at the point $x$ and $\mathbf{R}_{o}($.$) is the radiance of a surface patch$ with unit normal $\hat{\mathbf{n}}_{o}(x)$.

For simplification purposes, most algorithms assume that the surface has Lambertian reflectance. In this case, the reflectance map is the cosine of the angle between the unit vector $\hat{\mathbf{s}}_{o}$ in the light direction and the normal vector, $\hat{\mathbf{n}}_{o}$ :

$$
\mathbf{E}_{o}(x)=\mathbf{R}_{o}=\cos \angle\left(\hat{\mathbf{s}}_{o}, \hat{\mathbf{n}}_{o}\right)=\hat{\mathbf{s}}_{o} \cdot \hat{\mathbf{n}}_{o}
$$

which leads to the first PDE studied in the SFS literature:

$$
\mathbf{I}(x) \sqrt{1+|\nabla \mathbf{u}(x)|^{2}}+\hat{\mathbf{s}}_{o} \cdot(\nabla \mathbf{u}(x),-1)=0 .
$$

where $\mathbf{u}(x)$ is the surface height at point $x=(x, y)$ above some reference plane. Note that the image irradiance, $\mathbf{E}_{o}$, has been replaced by the measured image gray value, $\mathbf{I}$, by assuming a linear relationship between them and dropping the scaling factor.

It is worth mentioning that Equation (8) is not the most general equation of SFS, but indeed, it is the simplest. Under real world circumstances the surface materials are not Lambertian, and in many cases the camera and the light are not far away from the object.

\section{Experimental Results}

We are using an intra-oral camera to capture overlapped images of artificial jaws. In this stage, the camera is fixed in a certain position. We move the jaw while the camera is fixed to image its different overlapped regions. This guarantees that all the images are calibrated. Figure 1 illustrates different stages in our system. Feature points are extracted and matched for each pair of images in sequence using the ASIFT approach. Figure 2 demonstrates the matching process between overlapped images. The ASIFT results in a set of point matches which are almost correct except few outliers. The RANSAC as described above remove the outliers. This is very important in order to obtain successful registration process. The 
sequence of images is numbered from left to right according to the order of taking the images. The teeth regions themselves do not have much texture information to extract features. However the edges and the contrast between the teeth and the gum plays a great role in this process. Also, for the registration approach we described above, only 4 points are required to estimate the transformation. This makes the resulting points more than enough for the success of registration. Using the resulting point correspondences, a projective transformation is computed and applied to the source image to get an image for stitching. Sequential images are registered together in their order and sequence as described in Figure 3. They are all put together in a bigger size image to build a panorama for the scanned jaw. Sequences of images are shown in Figure 4 with the stitching results. After the stitching process, some differences appear in the panoramic image. We carry out some filtering and blending operations to remove these differences as demonstrated in Figure 5. This process is completely automatic and does not need any manual interaction. A very important note we have to mention here is that:Actually, if such a system is needed in clinic, we can not image the whole patient's jaw in one shot. The mouth environment and the specifications of the used intr-oral camera allows us only to image overlapped parts of the jaw. This is considered to be a strong motivation for developing our system. The resulting scenes are used with the SFS technique shown above to reconstruct a 3D surface. The reconstructed surfaces include teeth details which can not be obtained by applying the SFS approach in [ㅁ] (see Fig. 6). We can notice that the resulting surface using that approach is over-smoothed and hence upper details of teeth are smeared. Also, the transitions between teeth and gum looks unrealistic which emphasize the advantage of the technique used in this paper. More surface reconstruction results are demonstrated in Figure 7 for different artificial jaws which assures the robustness of our approach. The whole processing time starting from taking images and ending with surface reconstruction is less than 10 minutes. The 3D surface reconstruction execution time is less than 1 minute. This is a big advantage for our system which makes it practical and suitable for dental clinics. This will not make the patient open his mouth for a long time to get his/her jaw model.

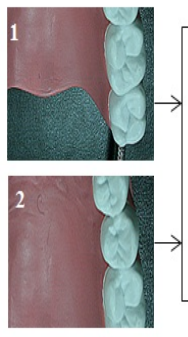

(a)

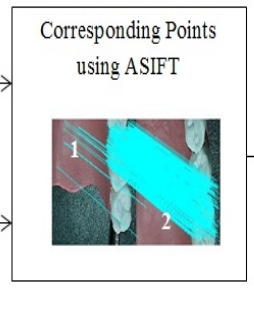

(b)

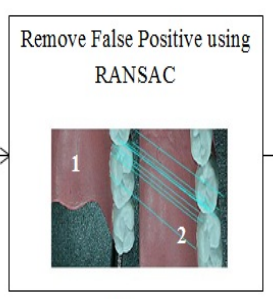

(c)

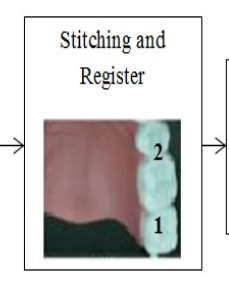

(d)

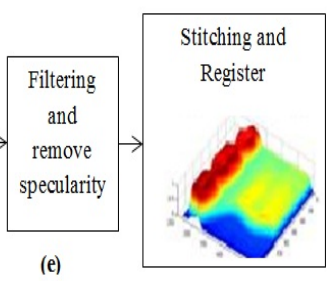

(f)

Figure 1: Two sequential overlapped images are given in (a) and used as inputs to the ASIFT algorithm to extract point correspondences demonstrated in (b). removing the outlier points using RANSAC in (c). The output points are used to register the two images as shown in (d). The resulting image is filtered in (e) to get a better image for the SFS as shown in (e). 


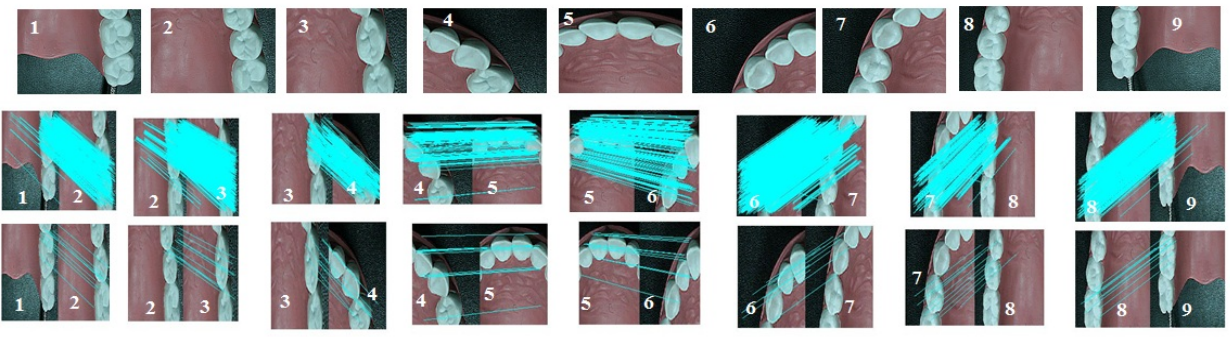

Figure 2: Different overlapped images and results of features matching: A sequence of overlapped images are illustrated in the first row and numbered from left to right. Point correspondences as a result of the ASIFT algorithm are illustrated in the second row. Point correspondences are demonstrated in the third row after removing the outliers matches using RANSAC.
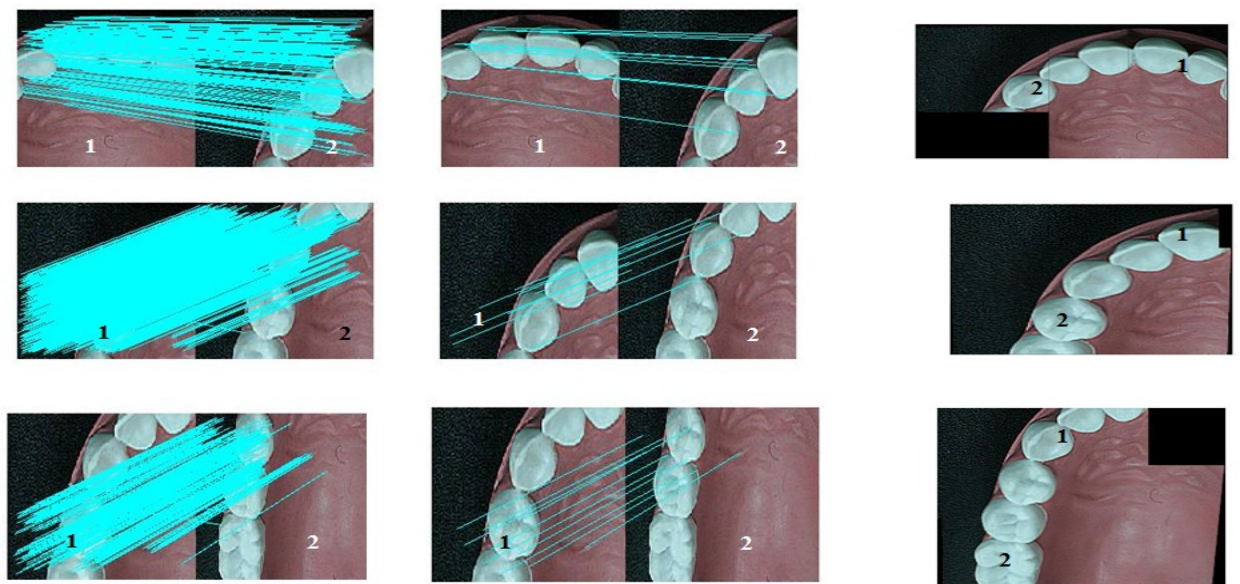

(a)

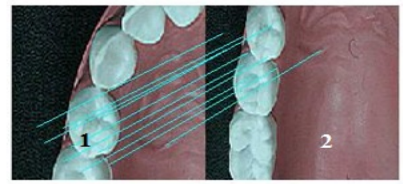

(b)

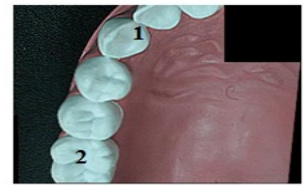

(c)

Figure 3: Three different examples to stitch two images together: Point correspondences are demonstrated in (a) using the ASIFT algorithm. Correspondences after removing the outliers using RANSAC are shown in (b). Registration of the two overlapped images is depicted in (c).

\section{Conclusions and Future Directions}

We have proposed an automatic approach for 3D surface reconstruction using calibrated images with the help of the ASIFT features matching. A calibrated camera is used to capture a sequence of images for artificial jaws. Images are stitched together by estimating projective transformations for minimizing the global differences between the scenes. The registration process requires accurate point correspondences which is computed using the ASIFT approach. Camera calibration parameters are involved in the formulation of modified version of the SFS technique to construct a 3D surface of the panoramic jaw image resulting for 

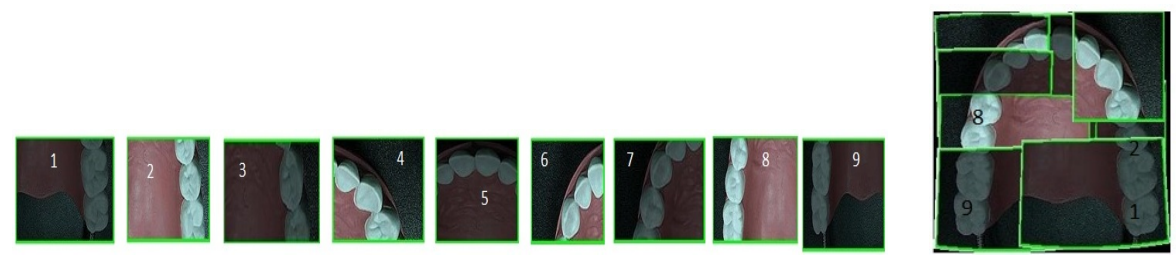

Figure 4: A jaw is imaged as 9 overlapped images illustrated from left to right. The stitching results (panoramic image) is shown at the last column where each sub-image is boxed by a green line to show the overlap between the views.
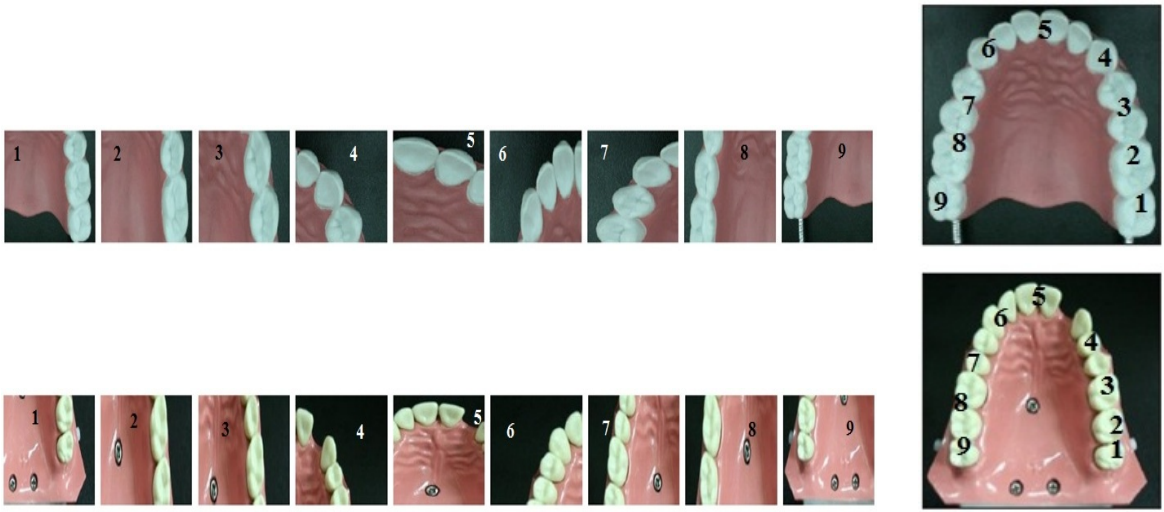

Figure 5: Two different jaws images panoramic reconstruction: A sequence of images is given for each case from left to right while the resulting panoramic image is given at the last column.

the stitching process. Our results are preferred to other approaches in the literature since fine teeth details can be reconstructed and the resulting surface is more realistic. Also the execution time is reasonable and practical in the sense of applying the proposed system in clinic. We plan to use flash laser cameras to construct exact surface and hence we can validate quantitatively and qualitatively our reconstructed surfaces. Also, when applying our system to real patients, the jaw centerline contour may be used to guide the stitching process (in case, the ASIFT features matching process completely fail). This contour will be estimated as a shape model computed from different real scans. Our preliminary centerline shape model is shown in Figure 8 for artificial jaw models.

\section{References}

[1] P. F. van der Stelt and S. M. Dunn, "3D-imaging in dental radiography," in Advances in Maxillofacial Imaging, A. G. Farman, ed., 367-372, 1997.

[2] R. L. Webber, R. A. Horton, D. A. Tyndall, and J. B. Ludlow, "Tuned-aperture computed tomography (TACT). theory and application for three-dimensional; dento-alveolar imaging," Dentomaxillofac. Radiol. 26:51-62, 1997.

[3] P. F. van der Stelt, S. M. Dunn, O. Tokuota, L. Jurgens, and J. H. Slater, "Comparison of two 

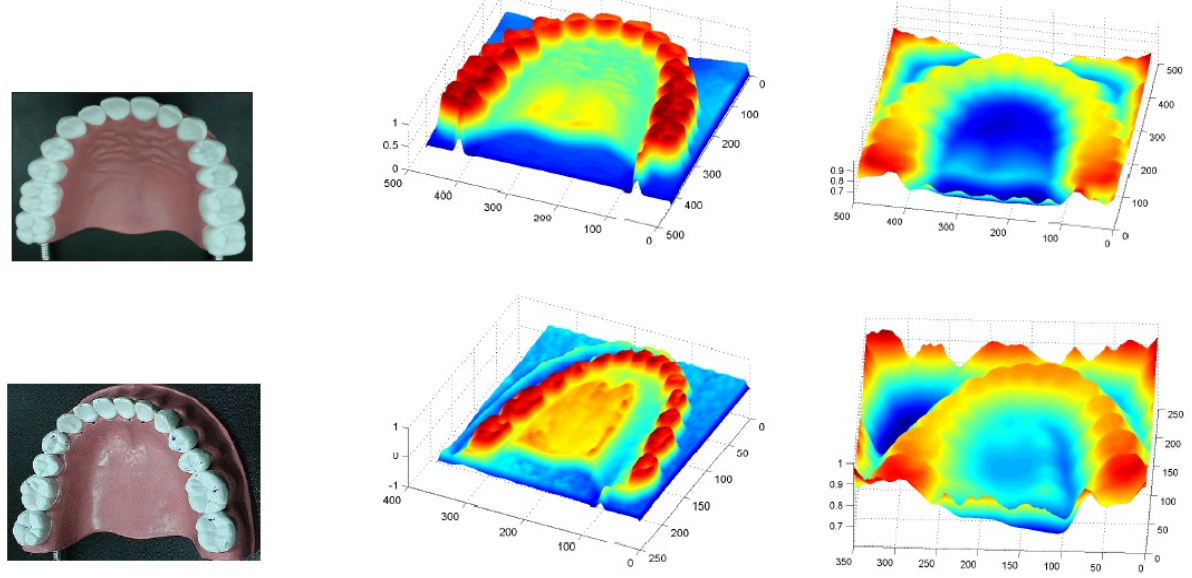

Figure 6: Two artificial jaws 3D surface reconstructions are demonstrated: the results of using our approach are shown in the middle and the results of the technique in [ $\mathrm{⿴囗}]$ ] are given in the right column.
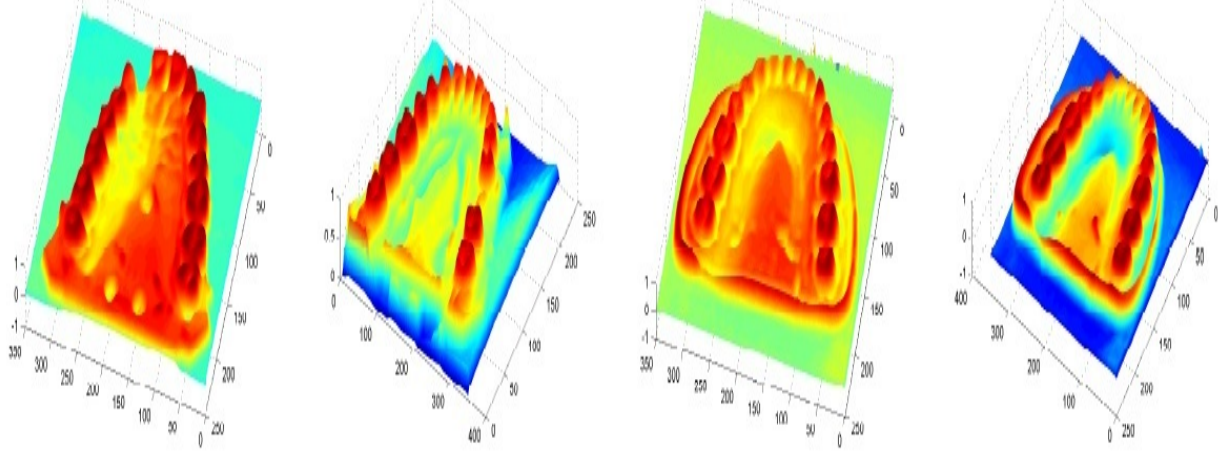

Figure 7: Different 3D surface reconstruction of artificial jaws.

localized CT- techniques for detecting periodontal angular bone defects," J. Dent. Res. 75(Special Issue), p. 128 (abstract 887), 1996.

[4] D. Laurendeau and D. Possart, "A computer-vision technique for the acquisition and processing of 3D profiles of dental imprints: An application in orthodontics," IEEE Transactions on Medical Imaging. 10:453-461, 1991.

[5] C. Bernard, A. Fournier, J. M. Brodeur, H. Naccache, and R. Guay, "Computerized diagnosis in orthodontics," Proc. 66th Gen. Session Int. Assoc. Dental Res. Montreal, 1988. 

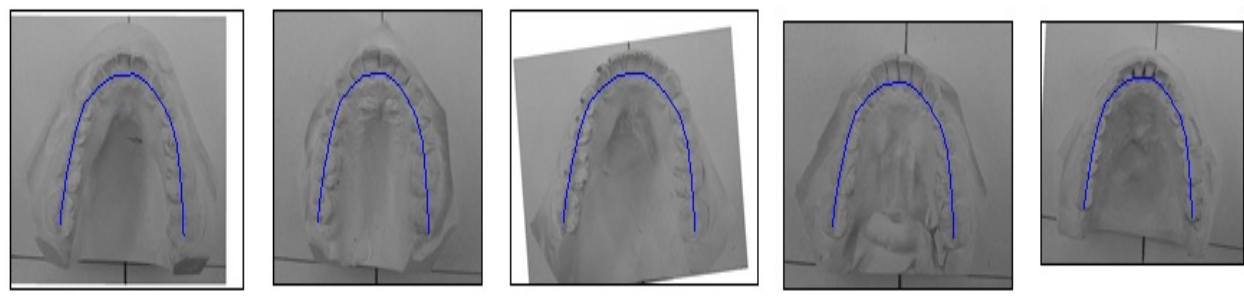

Figure 8: Illustration of different extracted jaw centerlines using the approach in [ $\square]$

[6] F. P. van der Linden, H. Boersma, T. Zelders, K. A. Peters, and J. H. Raben, “Three-dimensional analysis of dental casts by means of optocom,” J. Dent. Res. 51(4):1100, 1972.

[7] S. N. Bhatia and V. E. Harrison, "Operational performance of the traveling microscope in the measurement of dental casts, ” Br. J. Orthod. 14:147-1532, 1987.

[8] K. Tanaka, A. A. Lowe, and R. DeCou, "Operational performance of the re ex monograph and its applicability to the three-dimensional analysis of dental casts," Amer. J. Orthod. 83:304-305, 1983.

[9] A. A. Goshtasby, S. Nambala, W. G. deRijk, and S. D. Campbell, "A system for digital reconstruction of gypsum dental casts," IEEE Transactions on Medical Imaging. 16:664-674, Oct 1997.

[10] Richard Hartley and Andrew Zisserman , "Multiple View Geometry in computer vision,“ Cambridge University Press, 2003.

[11] J.M. Morel and G. Yu., "ASIFT: A New Framework for Fully Affine Invariant Image Comparison.," SIAM Journal on Imaging Sciences, , 438Ü469, 2009.

[12] M. A. Fischler and R. C. Bolles, "Random Sample Consensus, A Paradigm for Model Fitting with Applications to Image Analysis and Automated Cartography," In International Journal of Computer Vision, 24:381-395, 1981.

[13] B. K. P. Horn and M. J. Brooks, "Shape from Shading," Cambridge, Mass.: McGraw Hill, 1989

[14] K. Kimmel and A. Bruckstein, "Tracking level sets by level sets: A method for solving the shape from shading problem," Computer Vision and Image Understanding, 62(2):47-58, 1995.

[15] A. Pentland, "Extract Shape From Shading," Academic Press, MIT Media Lab, 2nd ed., 1988.

[16] P. S. Tsai and M. Shah, "A fast linear shape from shading," Proc. IEEE Computer Vision and Pattern Recog- nition Conf. (CVPR), Urbana, Illinois, 734-736, June 1992.

[17] J. K. Hasegawa and C. L. Tozzi, "Shape from shading with perspective projection and camera calibration," Computer and Graphics, 20(3):351-364, 1996.

[18] K. M. Lee and J. Kuo, "Shape from shading with generalized reflectance map model," Computer Vision and Image Understanding, 67:143-160, August 1997.

[19] G. Xu and Z. Zhang, "Epipolar Geometry in Stereo, Motion and Object Recognition," Kluwer Academic, The Netherlands, 1996. 
[20] N.Carter, J.Pusateri, D.Chen ,H.Ahmed, A.Farag, "Shape from Shading for Hybird Surfaces as Applied to Tooth Reconstruction,' International Conference on Image Processing ICIP'2010, 4049-4052, Sept. 2010.

[21] David A. Forsyth and Jean Ponce, Computer Vision: A Modern Approach, Pearson Education, New York, 2003.

[22] S. M. Yamany, A. A. Farag, E. Rickard, D. Tasman, and A. G. Farman. "A robust 3D reconstruction system for human jaw modeling," In Proceedings of the Second International Conference Medical Image Computing and Computer-Assisted Intervention (MICCAI), 778-787, Berlin, Germany, Springer-Verlag, 1999.

[23] M. Sabry Hassouna and A. A. Farag, "Robust Centerline Extraction Framework Using Level Sets," Proc. of IEEE Conference on Computer Vision and Pattern Recognition (CVPR), San Diego, CA, USA , 458-465 June 20-26, 2005,. 